\title{
PREVALENCIA DE COMPLICACIONES EN PACIENTES SOMETIDOS A CATÉTER, QUE INGRESARON A UNA UNIDAD DE HEMODIÁLISIS EN UN CENTRO HOSPITALARIO DE SANTO DOMINGO, REPÚBLICA DOMINICANA, DURANTE EL PERÍODO MARZO-OCTUBRE 2019
}

\author{
Prevalence of complications in patients undergoing catheters who \\ were admitted to a hemodialysis unit in a hospital in Santo Domingo, \\ Dominican Republic, during the period March-October 2019
}

\author{
Alfredo Polanco del Orbe ${ }^{a}$, Daniel Bisonób, Paola Hernández ${ }^{c}$, Bianna Matos ${ }^{d}$, \\ Jhonatan Rojas ${ }^{\mathrm{e}}$, Nirys L. Mateo Faxasf, Lorent Pérez Sotog, Erika P. Rosario Zorrilla ${ }^{\mathrm{h}}$ \\ y Leslie C. Valdez Polancoi
}

Recibido: 6 de octubre, 2019 • Aprobado: 4 de diciembre, 2019

Cómo citar: del Orbe AP, Bisonó D, Hernández P, Matos B, Rojas J, Mateo Faxas NL, Pérez Soto L, Rosario Zorrilla EP, Zorrilla R, Valdez Polanco LC. Prevalencia de complicaciones en pacientes sometidos a catéter, que ingresaron a una unidad de hemodiálisis en un centro hospitalario de Santo Domingo, República Dominicana, durante el período marzo-octubre 2019. cysa [Internet]. 16 de junio de 2020 [citado 17 de junio de 2020];4(2):81-88. Disponible en: https://revistas.intec.edu.do/index.php/cisa/article/view/1758

Resumen

Introducción: la insuficiencia renal crónica se define como la pérdida progresiva, generalmente irreversible, de la tasa de filtración glomerular, se traduce en un conjunto de síntomas y signos a los cuales se les denomina síndrome urémico y que en su estado terminal es incompatible con la vida.

Objetivo: determinar la prevalencia de complicaciones en pacientes sometidos a catéter que ingresaron a una unidad de hemodiálisis en un centro hospitalario de Santo Domingo, República Dominicana durante el periodo marzo-octubre 2019.

Metodología: estudio retro-prospectivo, descriptivo, de corte transversal y observacional en el que se analizaron las encuestas realizadas a 59 pacientes mediante un formulario en un centro hospitalario de Santo Domingo, República Dominicana.

\footnotetext{
a Nefrólogo, Hospital Traumatológico Dr. Ney Arias Lora. Docente de la cátedra de Nefrología del Instituto Tecnológico de Santo Domingo (INTEC), República Dominicana.

Correos-e: Alfredo.polanco@intec.edu.do,apolancodelorbe@hotmail.com

*Estudiantes de Medicina del lnstituto Tecnológico de Santo Domingo (INTEC).

b Correo-e: daniel.bisono@hotmail.com

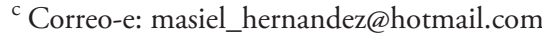

Introduction: Chronic renal failure is defined as the progressive loss, usually irreversible, of the glomerular filtration rate that results in a set of symptoms and signs called uremic syndrome, and that in their terminal state, is incompatible with lifetime.

Objective: To determine the prevalence of complications in patients undergoing catheterization who entered a hemodialysis unit in a hospital in Santo Domingo, Dominican Republic during the period March-October 2019.

Methodology: Retro-prospective, descriptive, cross-sectional study and observation in which the surveys of 59 patients were analyzed using a form in a hospital in Santo Domingo, Dominican Republic.

\footnotetext{
d Correo-e: biannajosefa@hotmail.com

e Correo-e: jhonatanrc792@gmail.com

${ }^{\mathrm{f}}$ Correo-e: mateofaxasniryslaura@hotmail.com

g Correo-e: lorentsoto@gmail.com

h Correo-e: erikarosario2103@gmail.com

i Correo-e: 1cvp0108@gmail.com
} 


\section{Alfredo Polanco del Orbe, Daniel Bisonó, Paola Hernández, Bianna Matos, Jhonatan Rojas, Nirys L, Mateo Faxas, Lorent Pérez Soto, Erika P. Rosario Zorrilla y Leslie C. Valdez Polanco}

Resultados: el $61 \%$ de los pacientes tuvo alguna complicación, donde predominaron las infecciones en un $56 \%$ de los casos, las mismas se observaron principalmente en aquellos pacientes a los que se les colocó de 5 a 6 catéteres.

Conclusiones: los pacientes de la unidad con catéter presentan un gran número de complicaciones, las cuales son proporcionales al número de catéteres colocados a los pacientes.

Recomendaciones: se debe promover la asistencia a consultas nefrológicas previa al inicio de hemodiálisis, para que de esta forma los pacientes ingresen a diálisis con fístula arteriovenosa funcional y obtener mejores resultados.

Palabras clave: catéter; hemodiálisis; insuficiencia renal crónica; complicaciones; nefrología.

\section{Introducción}

La insuficiencia renal crónica se define como la pérdida progresiva, generalmente irreversible, de la tasa de filtración glomerular, lo que se traduce en un conjunto de síntomas y signos a los cuales se le denominó síndrome urémico, y que en su estado terminal es incompatible con la vida. ${ }^{1}$ La insuficiencia renal crónica (IRC) es un síndrome con manifestaciones clínicas muy variadas que afecta a la mayor parte de órganos y sistemas (tabla 1), la misma es un reflejo de las funciones complejas que el riñón desempeña en condiciones fisiológicas, así como de las severas consecuencias que provoca la disfunción renal. ${ }^{2}$ Los pacientes con IRC presentan un perfil clínico general, con edades comprendidas entre 50-70 ańos, siendo el 58,2\% mujeres y el $41,8 \%$ hombres, con múltiples patologías asociadas a su enfermedad. ${ }^{3}$ Las causas más frecuentes de retirada de catéteres por complicaciones fueron: $75 \%$ por déficit de flujo sanguíneo, $11 \%$ por infección, $6 \%$ por pérdida de sutura, $3 \%$ por oclusión, $3 \%$ por acodamiento. ${ }^{4}$

El tratamiento de la insuficiencia renal crónica es la diálisis en sus dos modalidades, hemodiálisis
Results: $61 \%$ of the patients had some complication, where infections predominated in 56\% of the cases, observed mainly in patients who had 5 to 6 catheters placed in them.

Conclusions: Patients in the catheter unit have a large number of complications which are proportional to the number of catheters placed in them.

Recommendations: Attendance at nephrology consultations prior to the onset of hemodialysis should be promoted so that patients can enter dialysis with a functional arteriovenous fistula to obtain better results.

Keywords: Catheter, hemodialysis, chronic renal failure, complications, nephrology.

(HD), diálisis peritoneal, y el trasplante, siendo esta la más común, la depuración sanguínea extracorpórea, que va a sustituir parcialmente las funciones renales de excreción de solutos, regulación del equilibrio ácido-base y electrolítico, y eliminación de líquido retenido. La más utilizada es la hemodiálisis alcanzando un 80 a $90 \%$ y la diálisis peritoneal continua ambulatoria (DPCA) se utiliza en un 10 a $20 \%$, con algunas excepciones. ${ }^{1}$

El catéter como acceso para realizar el proceso de hemodiálisis, aunque no está recomendado por las guías K-DOQI y EPBG, cada vez tiene una mayor prevalencia en las salas de hemodiálisis. En la actualidad, los catéteres tunelizados con cuff de doble luz son de elección en la población en diálisis. La ventaja fundamental de estos es la facilidad con la que pueden ser insertados y que permiten su uso de manera inmediata. A pesar de que la técnica provee de múltiples ventajas, a esta se le atribuyen una serie de complicaciones que pueden dividirse en tempranas o tardías. Las complicaciones más frecuentes son las tardías y son la disfunción del catéter secundaria a procesos trombóticos o migración del catéter, la estenosis venosa central y la bacteriemia relacionada con el catéter (CRB). La trombosis intrínseca representa la principal compli- 
cación y la causa fundamental de pérdida del catéter. Debe intentarse tratamiento intraluminal con enzimas líticas y si son incapaces de restaurar el flujo se debe cambiar el catéter previa destrucción de la vaina de fibrina. La estenosis venosa central normalmente es asintomática. En las lesiones sintomáticas, la angioplastia percutánea es el tratamiento de elección. ${ }^{5}$

Las CRB con catéter central para hemodiálisis es una de las principales complicaciones de los pacientes. ${ }^{6}$ Se han desarrollado distintas medidas preventivas para disminuir la tasa de infección. La adopción de un protocolo de asepsia estricto reduce significativamente la incidencia de CRB. El tratamiento precoz es la herramienta más efectiva para prevenir el desenlace fatal que a veces ocurre. ${ }^{7}$

Los pacientes diagnosticados con insuficiencia renal han perdido la capacidad de realizar la filtración de la sangre a través de los riñones, razón por la cual deben ser tratados con terapia de sustitución de la función renal, mejor conocido como diálisis. Para la realización de este proceso es necesario crear un acceso vascular transitorio, es decir, la colocación de un catéter intravenoso hasta que el paciente sea candidato para la colocación de una fístula arteriovenosa que es el tipo de acceso ideal para realizar el proceso de diálisis.

Se estima que a la mayoría de los pacientes que ingresa a las unidades de hemodiálisis en los centros hospitalarios de Santo Domingo, República Dominicana, por primera vez, se les coloca un catéter para empezar el proceso de hemodiálisis. Se supone que esto es solo una solución ambulatoria, sin embargo, se suele extender su uso hasta un tiempo indefinido. Esto se ve relacionado a la desinformación del paciente en cuanto a su condición de salud, lo cual se asocia al hecho de que estos pacientes nunca asistieron a una consulta previa de nefrología. También se le atribuye a sus creencias, tabúes y al miedo popular de que al momento de ser dializado es más susceptible a fallecer. Debido a esto, es preciso demostrar que el uso prolongado del catéter impacta de forma negativa la calidad de vida del paciente y que se debe fomentar la asistencia a consulta antes de iniciar la hemodiálisis.

Ha surgido la necesidad de realizar esta investigación debido a que las unidades de hemodiálisis de Santo Domingo, en muchos casos, no ofrecen la mejor opción de técnicas para canalizar a los pacientes, que le brinden a largo plazo bienestar. En efecto, es propicio demostrar que los pacientes que ingresan a las unidades de hemodiálisis con catéter suelen tener mayor susceptibilidad a padecer de alguna complicación que los que ingresan con fístula arteriovenosa, por esto se considera oportuno que los centros de salud y hospitales tengan la capacidad de empezar a reconocer la importancia del acceso arteriovenoso y aplicarlo como método principal en las unidades de hemodiálisis de los hospitales de Santo Domingo, para de este modo garantizar que el paciente tenga una mejor calidad de vida.

\section{Material y métodos}

Este estudio fue de carácter retro-prospectivo, realizado durante el periodo marzo-octubre del 2019, tanto de fuentes primarias y secundarias de información; descriptivo puesto que los autores evaluaron la frecuencia y la tendencia de una población con respecto a una enfermedad; observacional, dado que los autores se limitaron a medir las variables sin ejercer control sobre el factor de estudio, detallando de modo sistemático todos los datos sin alteración alguna; y de corte transversal, pues las variables fueron medidas en el tiempo propuesto sin darle seguimiento después de haber finalizado la recolección de datos.

La información fue recopilada mediante dos métodos: un formulario de selección múltiple (ver anexo 1), el cual fue llenado por los pacientes de la unidad de hemodiálisis de un centro hospitalario de Santo Domingo, República Dominicana que cumplían con los criterios de inclusión y exclusión; el mismo 
fue realizado por los autores de la investigación, con este se determinó la prevalencia de complicaciones en pacientes sometidos a catéter; de igual forma, se utilizaron las historias clínicas de los pacientes de la unidad de hemodiálisis de un centro hospitalario de Santo Domingo, República Dominicana, las cuales se usaron para evaluar qué cantidad de pacientes acudieron a una consulta previa de nefrología antes de iniciar la hemodiálisis, el sexo más frecuente en la colocación de catéter, y qué pacientes, a los cuales se les colocó catéter, presentaron algún tipo de complicación relacionada con el mismo, por ende, cuántos cambios de catéter han necesitado.

En total se evaluaron 59 casos debido a que este es el número de pacientes de la unidad hemodiálisis de un centro hospitalario de Santo Domingo, República Dominicana, que cumplía con los criterios de inclusión y exclusión de la investigación.

\section{Resultados}

Gráfica 1. Prevalencia de complicaciones en pacientes sometidos a catéter en una unidad de hemodiálisis. $(\mathrm{N}=59)$

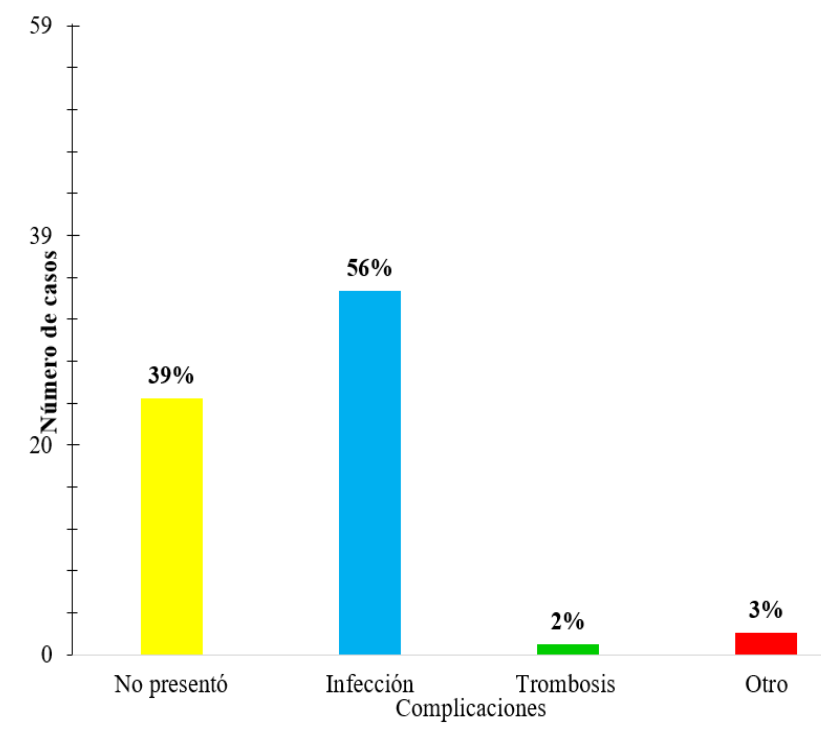

Fuente: Polanco, Faxas, Pérez, Rosario y Valdez, (2019). BioINTEC Jornada 83.
Para la realización de este gráfico se tomaron en cuenta los pacientes que habían presentado más de una complicación relacionada con el uso de catéter. De acuerdo con los resultados obtenidos, la mayor cantidad de pacientes encuestados que ingresaron a diálisis con catéter presentaron una infección con un $56 \%$, correspondiendo a 34 de los casos. Mientras que hubo un $39 \%$ de los pacientes que no presentaron ningún tipo de complicación relacionada al uso de catéter, representando 24 casos. Un $2 \%$ de los pacientes ingresados con catéter presentó casos de trombosis venosa, y un $3 \%$ presentó otro tipo de complicación no especificada.

Gráfica 2. Porcentaje de pacientes que ingresaron a la unidad por situaciones de emergencia. $(\mathrm{N}=59)$

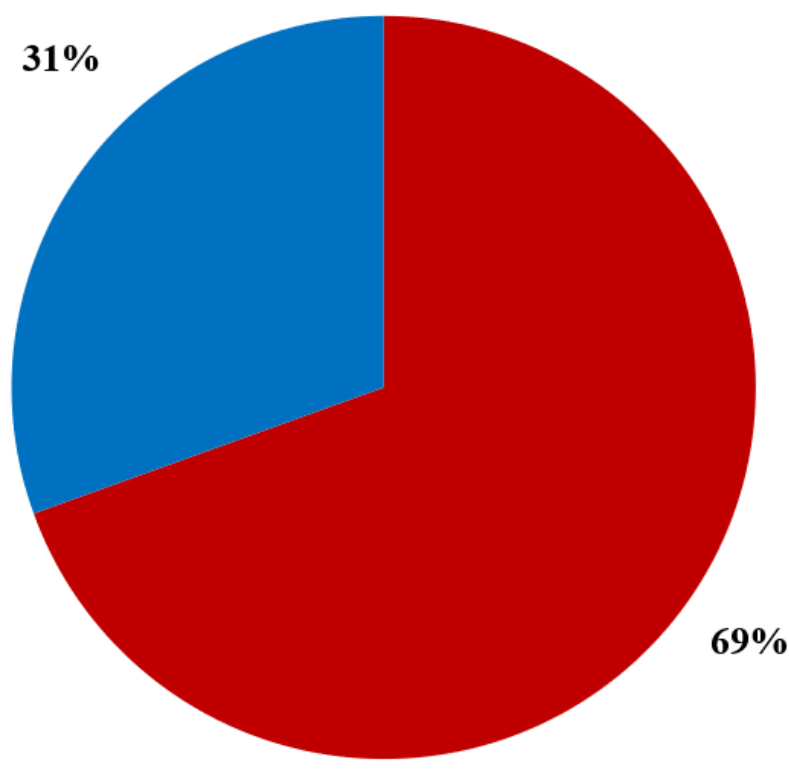

$$
\begin{array}{|l|}
\text { - Situación de emergencia } \\
\text { Indicaciones de su médico }
\end{array}
$$

Fuente: Polanco et al., (2019). BioINTEC 83. 
Los datos recolectados demuestran que un $69 \%$ de los pacientes encuestados que ingresaron a una unidad de hemodiálisis fue debido a una situación de emergencia, correspondiendo a 41 de los casos. También manifiestan que un $31 \%$ de los pacientes encuestados que ingresaron a una unidad de hemodiálisis fue por indicaciones de su médico especialista, correspondiendo 18 de los casos.

Gráfica 3. Distribución de pacientes con catéter según el sexo. $(\mathrm{N}=59)$

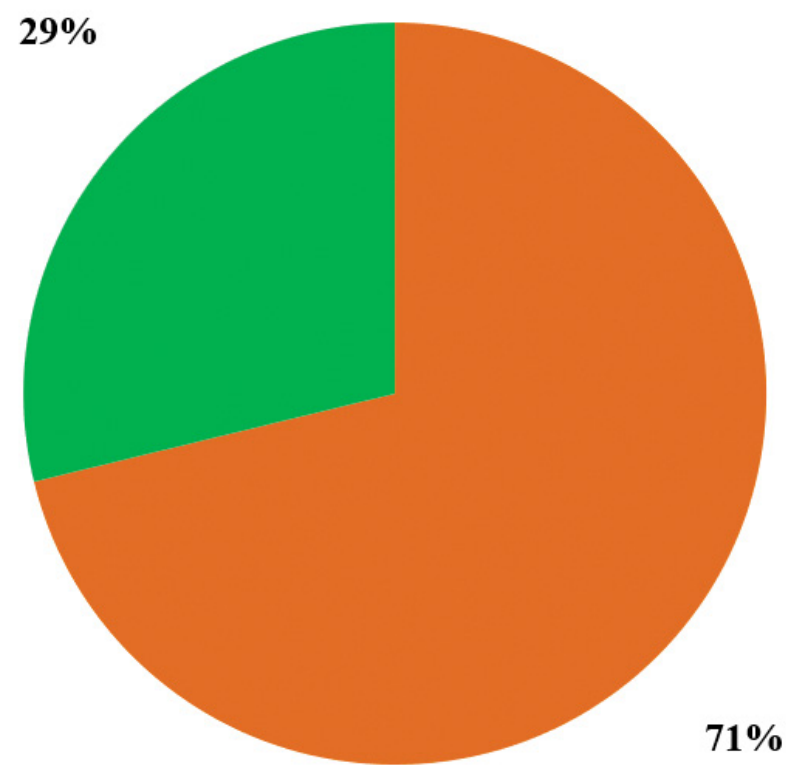

Fuente: Polanco et al., (2019). BioINTEC Jornada 83.

Los resultados demuestran que la mayoría de los pacientes encuestados pertenecían al sexo masculino, correspondiendo a 42 de los casos, mientras que solo 17 de los casos pertenecían al sexo femenino.
Gráfica 4. Número de cambios de catéteres realizados a los pacientes. $(\mathrm{N}=59)$

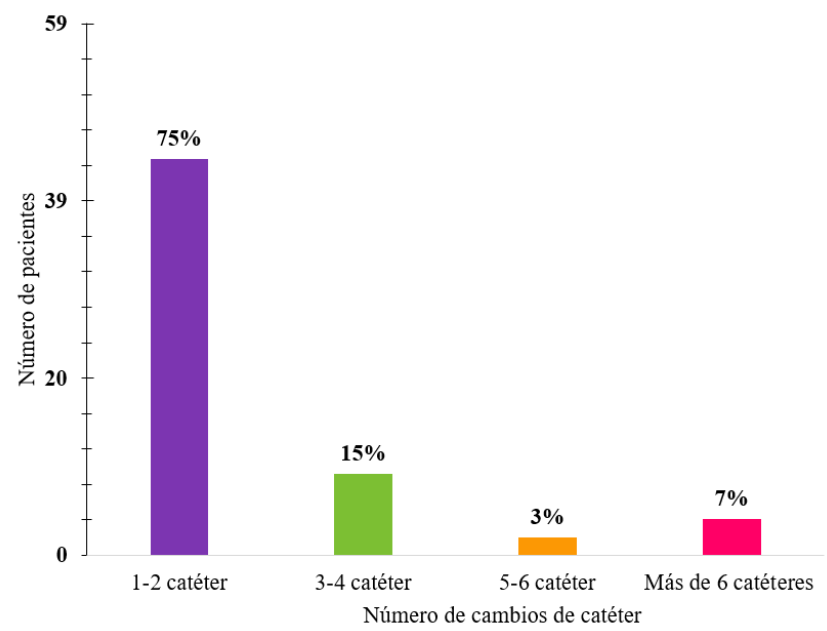

Fuente: Polanco et al., (2019). BioINTEC Jornada 83.

De acuerdo con los resultados obtenidos, la mayor cantidad de pacientes encuestados que ingresaron a diálisis con catéter han tenido entre uno y dos cambios de catéter, lo que representa $75 \%$, correspondiendo a 44 de los casos. Mientras que hubo un $15 \%$ de los pacientes que han tenido que realizarse entre tres y cuatro cambios de catéter, correspondiendo a 9 casos. A un $3 \%$ de los pacientes ingresados con catéter se le ha realizado de cinco a seis cambios, y a un $7 \%$ más de seis cambios de catéter.

Tabla 1. Proporción entre el número de cambios de catéteres realizados a los pacientes de la unidad de hemodiálisis y el número de complicaciones presentadas. $(\mathrm{N}=59)$

\begin{tabular}{|c|c|c|c|}
\hline $\begin{array}{c}\text { Número de } \\
\text { cambios de } \\
\text { catéter }\end{array}$ & $\begin{array}{c}\text { Número } \\
\text { de } \\
\text { pacientes }\end{array}$ & $\begin{array}{c}\text { Cantidad de } \\
\text { complicaciones } \\
\text { presentadas }\end{array}$ & $\begin{array}{c}\text { Porcentaje de } \\
\text { complicaciones }\end{array}$ \\
\hline $1-2$ catéter & 44 & 20 & $\mathbf{4 5 \%}$ \\
\hline $3-4$ catéter & 9 & 8 & $\mathbf{8 8 \%}$ \\
\hline 5-6 catéter & 2 & 1 & $\mathbf{5 0 \%}$ \\
\hline $\begin{array}{c}\text { Más de 6 } \\
\text { catéteres }\end{array}$ & 4 & 4 & $\mathbf{1 0 0 \%}$ \\
\hline
\end{tabular}

Fuente: Polanco et al., (2019). BioINTEC Jornada 83. 
La tabla siguiente presenta la proporción entre el número de catéteres colocados a los pacientes pertenecientes a una unidad de hemodiálisis y el número de complicaciones que presentaron. De los pacientes evaluados, el grupo que había tenido más de 6 catéteres en $100 \%$ de los casos presentaron algún tipo de complicación. Los pacientes que se les realizó de 5-6 cambios de catéter en un 50\% de los casos presentaron algún tipo de complicación, mientras que los pacientes que se les realizó de 3-4 cambios presentaron complicaciones en un $88 \%$. El grupo con menos porcentaje de complicaciones fue el que solamente habían tenido de 1-2 cambios de catéter, con un $45 \%$.

\section{Discusiones y conclusiones}

La prevalencia encontrada en el período de análisis, en cuanto a la infección asociada al uso del catéter intravascular de hemodiálisis fue de un $56 \%$ (gráfica 1). Según un estudio realizado en el periodo 20152016, publicado en la Revista Colombiana de Nefrología, titulado "Prevalencia de infección asociada a catéter de hemodiálisis en el Hospital Universitario Clínica San Rafael"', de la Dra. Maité Hurtado et al., la prevalencia de infección asociada a catéter fue de 5,62\%. Se incluyeron 320 pacientes en total, 18 con infección asociada a catéter, lo cual no es igual al resultado que se obtuvo en la presente investigación. Esto puede deberse a que el número de la muestra no fue el mismo y que ambas fueron realizadas en países distintos, con calidades de vida diferentes. No obstante, se puede observar que el porcentaje de pacientes con complicaciones fue muy elevado. Otra diferencia, de los pacientes con infección asociada a dispositivo de hemodiálisis, el 26,82\% fue constituido por diabéticos versus el 38,8 \% sin dicho diagnóstico, mientras que en la presente investigación fue constituida por un $29 \%$ diabetes, $54 \%$ hipertensión arterial y un $17 \%$ otros (anexo 2).
Estudios anteriores demuestran que los pacientes hemodializados con catéter siempre presentaron complicaciones durante el proceso, mismas que evidencian un deterioro en su calidad de vida. ${ }^{8}$

Los hombres son el sexo más frecuente en el uso del catéter, con un porcentaje de $71 \%$ (gráfica 3), lo que se asemeja con el resultado de Hurtado $^{8}$ en donde el 66,67 \% del Grupo 1 estaba constituido por hombres; mientras que el 60,93\% correspondientes al Grupo 2 eran mujeres.

El número de pacientes que ingresan a plan de hemodiálisis con un catéter está relacionado con la consulta previa al inicio de la hemodiálisis. En la investigación se encontró que el 31 \% asistió a consulta previa al plan de hemodiálisis, mientras que el $69 \%$ ingresó por situación de emergencia. Por lo que se puede concluir que ambas variables son directamente proporcionales, es decir, una dependerá de la otra.

De los pacientes que presentaron complicaciones asociadas al uso de catéter, el $75 \%$ recibió dos cambios de catéter, un $15 \%$ de tres a cuatro cambios, un $3 \%$ de cinco a seis cambios (gráfica 4). Al realizar una relación entre el número de cambios y el número de complicaciones presentadas por paciente se determinó que los pacientes que tuvieron 6 o más cambios todos presentaron complicaciones, en los pacientes de cinco a seis cambios solo un medio presentó complicaciones, los que se realizaron tres a cuatro cambios, un $88 \%$ presentó complicaciones y el grupo con menos porcentaje de complicaciones fue el que solo se hizo de uno a dos cambios de catéter (tabla 1). Lo que demostró que ambas variables son dependientes; el número de catéter colocados se ve influenciado por las complicaciones que presente el paciente. 


\section{Bibliografía}

1. Torres Zamudio C. Insuficiencia renal crónica. Rev Med Hered. 2003;14(1):1-4. Disponible en: http://www.scielo.org.pe/scielo.php?script=sci_ arttext\&pid=S1018-130X2003000100001\&lng=es.

2. López Revuelta K, Portoles J, Fernández-Juárez G. Present of the electronic clinical record in outpatient nephrology. Nefrologia: publicación oficial de la Sociedad Espanola Nefrologia [Internet]. 2006. Disponible en: https://www. researchgate.net/profile/Jose_Portoles2/publication/6361428_Present_of_the_electronic_ clinical_record_in_outpatient_nephrology/ links/5523e6bb0cf24f160943bbe9.pdf\# page $=65 \% 20$ [Accessed $\% 2011 \% 20$ Oct. $\% 20$ 2019].

3. Galache A. Diagnósticos de enfermería en pacientes con insuficiencia renal crónica en hemodiálisis. Rev Soc Esp Enferm Nefrol. 2004;7(3):158-63. Disponible en: http://scielo. isciii.es/scielo.php?

4. Crespo R, Contreras M, Casas R, Muñoz I, Moreno M, Suanes L. Estudio retrospectivo de las complicaciones de los catéteres temporales para hemodiálisis. Rev Soc Esp Enferm Nefrol. 2011;14(1):43-9. Disponible en: http://scielo. isciii.es/scielo.php?script=sci_arttext\&pid= S1139-13752011000100007\&lng=es.

5. Muñiz-Gómez M. Catéteres venosos centrales como acceso para hemodiálisis crónica. Diálisis y trasplante. 2013;24(1):23-7. Disponible en: https://www.elsevier.es/es-revista-dialisis-trasplante-275-articulo-central-venous-catheters-as-access-S1886284511002001
6. Gruss E, Corchete E. El catéter venoso central para hemodiálisis y su repercusión en la morbimortalidad. Nefrología. 2012;3(6). Disponible en: https://www.revistanefrologia.com/ es-el-cateter-venoso-central-hemodialisis-articulo-X2013757512001460

7. Múniziz Gómez M. Complicaciones de los catéteres venosos para hemodiálisis. Elsevier 2011;32(3):123-4. Disponible en: https://www. elsevier.es/es-revista-dialisis-trasplante-275-articulo-complicaciones-de-los-cateteres-venosos-S188628451100110X

8. Gómez J, Pimienta L, Pino R, Hurtado M, Villaveces M. Prevalencia de infección asociada a catéter de hemodiálisis en el Hospital Universitario Clínica San Rafael. Rev. colom. nefrol. 2018;5(1):17-25. Disponible en: http://www. scielo.org.co/scielo.php?script=sci_arttext $\&$ pid $=S 2500-50062018000100017 \& \operatorname{lng}=$ en . http://dx.doi.org/10.22265/acnef.5.2.283.

9. Sánchez-García A, Zavala-Méndez M, Pérez-Pérez A. Hemodiálisis: proceso no exento de complicaciones. San Luis Potosí, [Unidad Académica Multidisciplinaria Zona Media] México; 2012.

10. Franco Pérez N, Rodríguez Hung $S$, Telemaque $H$. Behavior of the arteriovenous fistulae for hemodialysis in patients with chronic renal failure. Rev Cubana Angiol Cir Vasc. 2015;16(1):3-8. Available at: http://scielo.sld.cu/scielo.php?script= sci_arttext\&pid=S1682-00372015000100002\& $\operatorname{lng}=$ es.

11. Ahmed GM, Mansour MO, Elfatih M, Khalid KE, Mohammed ME. Resultados de la fístula arteriovenosa para hemodiálisis en pacientes sudaneses: experiencia en un solo centro. Saudi J Kidney Dis Transpl. 2012;23(1):152-7. 
12. Cajuste Sequeira F, Sánchez Basurto M, Guillermo Corpus G, Peña Rodríguez J, Ramos Gordillo J. Sobrevida funcional de las fístulas arteriovenosas comparadas con los catéteres tunelizados en pacientes en hemodiálisis crónica. Acta Médica Grupo Ángeles. 2018;16(4):310-5. Disponible en: https://www.medigraphic.com/ pdfs/actmed/am-2018/am184f.pdf

13. Adell Lleixà M, Casadó Marin L, Andújar Solé J, Solà Miravete E, Martínez Segura E, Salvadó Usach T. Valoración de la calidad de vida relacionada con la salud en la enfermedad renal crónica terminal mediante un cuestionario de resultados percibidos por los pacientes. Enferm Nefrol. 2016;19(4):331-40. Disponible en: http://scielo.isciii.es/scielo.php?script=sci_ arttext\&pid=S2254-28842016000400004\& $\operatorname{lng}=\mathrm{es}$.

14. Valdivia Arencibia J, Gutiérrez Gutiérrez C, Delgado Almora E, Méndez Felipe D, Treto Ramírez J, Fernández Madero I. Epidemiología de la enfermedad renal crónica y los factores de riesgo relacionados con la supervivencia. Investigaciones Medicoquirúrgicas 2012;3(1):10. Disponible en: http://www.revcimeq.sld.cu/ index.php/imq/article/view/43 